\title{
Development of a next-generation NIL library in Arabidopsis thaliana for dissecting complex traits
}

\author{
Richard S Fletcher ${ }^{1,2}$, Jack L Mullen ${ }^{1}$, Seth Yoder ${ }^{1}$, William L Bauerle ${ }^{3}$, Gretchen Reuning ${ }^{3}$, Saunak Sen ${ }^{4}$, Eli Meyer ${ }^{5}$, \\ Thomas E Juenger ${ }^{6}$ and John K McKay ${ }^{1 *}$
}

\begin{abstract}
Background: The identification of the loci and specific alleles underlying variation in quantitative traits is an important goal for evolutionary biologists and breeders. Despite major advancements in genomics technology, moving from QTL to causal alleles remains a major challenge in genetics research. Near-isogenic lines are the ideal raw material for QTL validation, refinement of QTL location and, ultimately, gene discovery.

Results: In this study, a population of 75 Arabidopsis thaliana near-isogenic lines was developed from an existing recombinant inbred line (RIL) population derived from a cross between physiologically divergent accessions Kas-1 and Tsu-1. First, a novel algorithm was developed to utilize genome-wide marker data in selecting RILs fully isogenic to Kas-1 for a single chromosome. Seven such RILs were used in 2 generations of crossing to Tsu-1 to create BC1 seed. BC1 plants were genotyped with SSR markers so that lines could be selected that carried Kas-1 introgressions, resulting in a population carrying chromosomal introgressions spanning the genome. BC1 lines were genotyped with 48 genome-wide SSRs to identify lines with a targeted Kas-1 introgression and the fewest genomic introgressions elsewhere. 75 such lines were selected and genotyped at an additional 41 SNP loci and another 930 tags using 2b-RAD genotyping by sequencing. The final population carried an average of 1.35 homozygous and 2.49 heterozygous introgressions per line with average introgression sizes of 5.32 and $5.16 \mathrm{Mb}$, respectively. In a simple case study, we demonstrate the advantage of maintaining heterozygotes in our library whereby fine-mapping efforts are conducted simply by self-pollination. Crossovers in the heterozygous interval during this single selfing generation break the introgression into smaller, homozygous fragments (sub-NILs). Additionally, we utilize a homozygous NIL for validation of a QTL underlying stomatal conductance, a low heritability trait.

Conclusions: The present results introduce a new and valuable resource to the Brassicaceae research community that enables rapid fine-mapping of candidate loci in parallel with QTL validation. These attributes along with dense marker coverage and genome-wide chromosomal introgressions make this population an ideal starting point for discovery of genes underlying important complex traits of agricultural and ecological significance.
\end{abstract}

Keywords: 2b-RAD, Fine-mapping, Quantitative trait loci, Stomatal conductance

\section{Background}

Linkage mapping of QTL is a common statistical approach in plant genetics where recombinant populations generated from crosses between inbred parent lines are used, in combination with molecular markers, to identify loci associated with variation in continuously distributed traits [1-8]. Mapping populations common to QTL analyses are many and include doubled haploids (DH), F2, backcross, advanced intercross,

\footnotetext{
* Correspondence: jkmckay@colostate.edu

'Department of Bioagricultural Sciences \& Pest Management, Colorado State University, 80523 Fort Collins, CO, USA

Full list of author information is available at the end of the article
}

nested association mapping and RILs. Mapping QTL for complex traits is now routine, with the typical output being QTL spanning large confidence intervals encompassing many (hundreds or more) possible causal genes [9].

The steps following QTL identification frequently involve functional validation of the QTL, and refinement of location (fine-mapping) towards the goal of identification of a causal gene - the major challenge in quantitative genetics today [10]. One of the most common approaches for accomplishing these objectives is through the development and phenotypic characterization of NILs [11]. The generation and phenotyping of NILs is considered a laborious 
and time consuming process, but the robust design leads to a minimal false positive rate.

NILs are lines containing a single or small number of genomic introgressions from a donor parent in a different and otherwise homogeneous genomic background. By homogenizing all genetic factors outside of the focal genomic region, the true effect of the QTL on the phenotype can be estimated relative to the line into which the introgression was introduced (i.e. void of the chromosomal introgression) [12]. In addition to the simplification of genetic analyses, NILs are considered genetically 'immortal' [13] which allows for replicated experiments across multiple environments resulting in more accurate estimates of effect size for complex traits. NILs have proven to be an effective resource for QTL validation and a logical starting point for the creation of fine-mapping populations [14-21].

Creation of a single near-isogenic line generally starts by crossing a line carrying the targeted QTL region to one of the parental lines of the population, thus creating a backcross population. Genome-wide genotyping of the backcross progeny is performed to identify recombination events allowing for selection of progeny which carry the target chromosomal introgression derived from the donor and recurrent parent genome elsewhere. Subsequent generations of self-pollination (selfing) are normally required to achieve homozygosity of the introgressed region and the process can take several backcrossing cycles to produce a NIL carrying an introgression of acceptable size and genomic location. An alternative approach has been the use of heterogeneous inbred families (HIFs) where NILs are selected from incompletely inbred lines which still harbour a small amount of heterozygosity at random intervals across the genome [22,23]. Analysis of a HIF population with molecular markers allows for the selection of lines heterozygous at a candidate genomic location, which in combination with further selfing and genotyping, enables selection of NILs derived from several heterogeneous genetic backgrounds. Producing NILs with smaller introgressions requires greater effort. Large populations are needed to break up small chromosomal segments, and high-density genotyping is required to discover them.

A NIL library is a family of near-isogenic lines where each line carries a different donor parent fragment and the population carries introgressions spanning the entire genome [24]. A NIL library is an ideal starting point for QTL validation, especially in cases where the library is derived from parent lines for which an immortal recombinant population (i.e. RILs, DH, etc.) already exists. In this case, QTL identified via traditional linkage mapping experiments performed on the mapping population can be immediately tested by selecting NIL(s) representing the QTL introgression and testing them for a phenotypic effect relative to the wild type recurrent parent. NIL libraries are also valuable starting material for fine-mapping QTL through the creation of sub-NILs [25], recombinant lines in which the original NIL introgression is broken into smaller genomic fragments. In this case, a candidate NIL is backcrossed to the recurrent parent and the progeny are genotyped using markers specific to the introgression region so that individuals carrying genomic fragments spanning the length of the original introgression can be identified. Subsequent phenotyping of the sub-NILs provides finer resolution of the region controlling the trait of interest, effectively narrowing the list of possible causal genes.

Several NIL populations are currently available to the Arabidopsis research community. Koumproglou et al. [26], using 31 simple sequence repeat (SSR) markers, created a population of Chromosome Substitution Strains by replacing chromosomes from the accession Columbia (Col-0) with homologous chromosomes from the accessions Landsberg erecta (Ler) and Niederzenz (Nd). Additionally, a population of more traditional NILs were created in a systematic approach where increasing lengths of chromosomal introgressions were introduced from Ler into the Col-0 background. Keurentjes et al. [27] generated a population of 92 NILs carrying genome-wide chromosomal introgression from the accession Cape Verde Islands (Cvi) into the Ler background. Selections were made from the genotyped RIL mapping population described by AlonsoBlanco et al. [28] and used in backcrosses to create the NIL library. The RIL population has been mapped for QTL underlying flowering time and carbon isotope ratio $\left(\delta^{13} \mathrm{C}\right)$ [29], recombination frequency [30], seed germination [31], seed mineral concentration [32] and fructose sensitivity [33]. The same 321 AFLP (Amplified Fragment Length Polymorphism) markers used to build the RIL map were used in the NIL breeding scheme. Finally, Torjek et al. [34] created a population of 140 reciprocal NILs from the accessions Col-0 and C24. (78 NILs in the Col-0 background and 62 lines in the C24 background) utilizing a total of 125 markers [35]. This NIL library has been used in subsequent studies of epistasis [36] and heterosis [37].

Here we report the development of a new population of 75 NILs constituting genome-wide chromosomal introgressions. The NIL population exploited inbred lines selected from the RIL population described in McKay et al. [38] as the starting material for backcrossing. Briefly, the RIL population is derived from a cross between the A. thaliana ecotypes Tsu-1 (CS1640), an accession originating from Tsushima, Japan and Kas-1 (CS903), an accession originating from Kashmir. These sites of collection are among the wettest and driest habitats, respectively, in the $A$. thaliana species range and the accessions differ in several aspects of drought physiology [39,40]. Recombinant populations derived from these diverse accessions will therefore segregate alleles underlying variation in these physiological traits, providing a powerful resource for identifying functional genes. 
We developed a population of 75 Arabidopsis thaliana NILs containing both homozygous and heterozygous introgressions, enabling simultaneous pursuit of QTL validation and fine-mapping. Genotyping the population with over 1,000 molecular markers has provided us with excellent resolution on the total number of introgressions existing in each NIL as well as their location and length. It is the most densely genotyped NIL population developed thus far by more than 3-fold. The utility of the NIL library is demonstrated in a simple case study where, in a single generation, we utilize a homozygous NIL to validate and localize a QTL for a low heritability physiological trait ( $g_{0}$; night-time stomatal conductance) while concurrently selfing heterozygous selections to create sub-NILs for further fine-mapping.

\section{Results}

\section{Marker-assisted NIL breeding program}

Figure 1 shows the breeding design for the NIL library. An algorithm was developed [see Additional file 1] to select RILs homozygous for Kas-1 alleles across one of each of the 5 Arabidopsis chromosomes. The results found 7 such RILs from the population of 346. These RILs were crossed to Tsu-1 and progeny were genotyped to confirm they were truly F1s. These were then crossed back to Tsu-1, creating $25 \mathrm{BC} 1$ families. Plants from each $\mathrm{BC} 1$ family were genotyped at the chromosome of interest to select individuals carrying Kas-1 alleles so they could be self-pollinated to generate BC1S1 seed. BC1S1 plants were genotyped using 48 genome-wide SSRs described in McKay et al. [38]. These data were analyzed using an algorithm [see Additional file 2] designed to identify a subset of lines representing Kas-1 chromosomal introgressions spanning the genome in otherwise Tsu-1 backgrounds. The algorithm was used to select 103 BC1S1 plants which were screened at an additional 149 single nucleotide polymorphisms (SNPs) loci using the Sequenom MassARRAY ${ }^{\odot}$ (Sequenom, San Diego, CA). Only 41 of 149 SNPs were informative for the parental lines. Finally, an additional 930 polymorphic loci were revealed using $2 \mathrm{~b}-\mathrm{RAD}$ [41] whereby genome complexity is reduced using class IIB restriction enzymes followed by sequencing on the SOLiD platform (Applied Biosystems, Foster City, CA).

\section{Polymorphisms detected between Tsu- 1 and Kas- 1 by 2b-RAD genotyping}

Restriction site-associated DNA (RAD) tag sequencing reduces genome complexity by focusing only on DNA

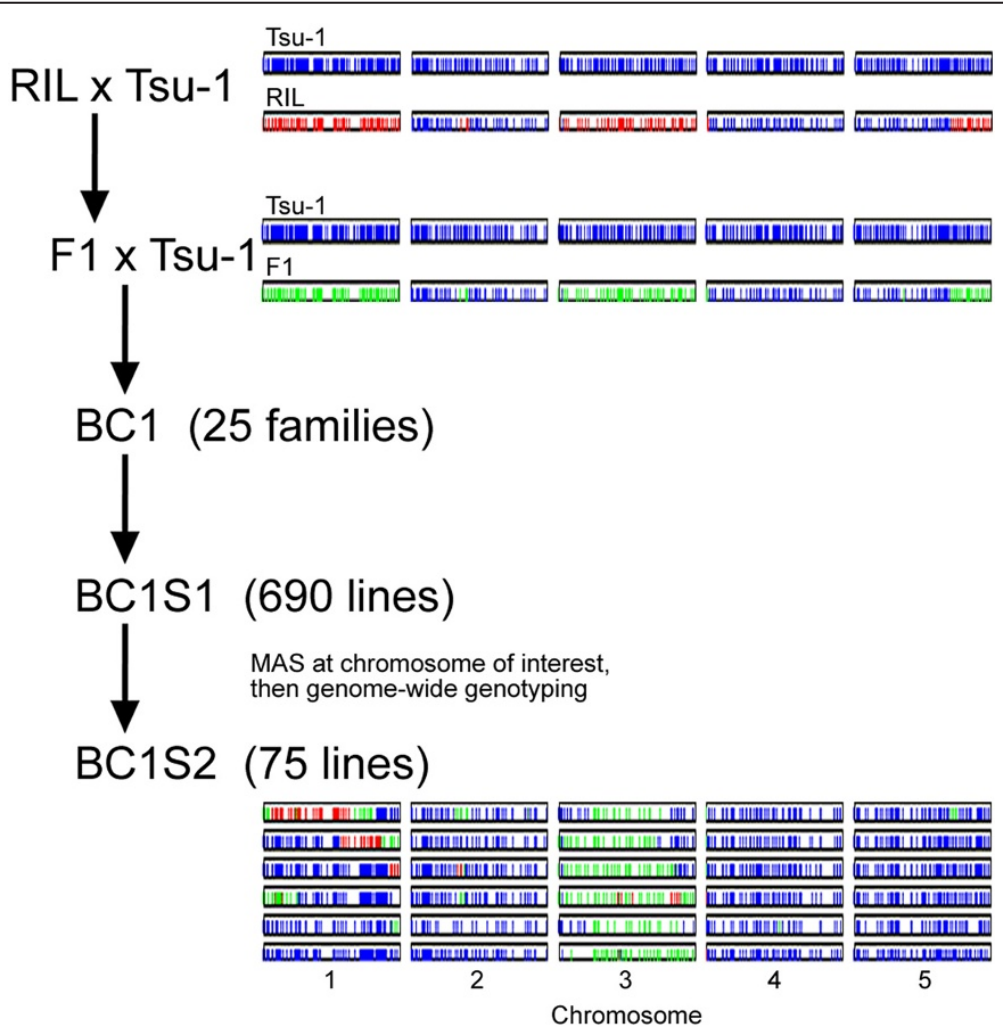

Figure 1 Breeding scheme of the NIL library. Breeding scheme and graphical genotypes of a set of NILs containing both homozygous introgressions (Chromosome 1) and heterozygous introgressions (Chromosome 3) derived from a single RIL. Each diploid breeding line is represented by a single row of 5 chromosomes where red coloring represents Kas-1 genotypes; Blue, Tsu-1; Green, heterozygous. Graphical genotypes of 6 of the 75 lines are shown. 
flanking the recognition sites of the selected restriction endonuclease [42]. The RAD method used in this study, described in [41] is a simple and effective means of discovering a large number of SNPs unique to the study population, avoiding the ascertainment bias associated with SNPs discovered via population surveys [43]. The 2b-RAD method utilizes the type IIB restriction enzyme, AlfI, which operates by cleaving DNA both upstream and downstream of the recognition site. The resulting tags are uniform in length, making them ideal for amplification and sequencing on next-generation platforms. Following digestion, tags were labelled with samplespecific oligonucleotide barcodes for multiplexed sequencing. Finally, reads were quality filtered and aligned to a collection of AlfI sites in the Col-0 Arabidopsis reference genome (TAIR9) in order to assign a physical location to each SNP.

Initially, 1319 polymorphisms were identified between the parent lines Tsu-1 and Kas-1 based on the 2b-RAD tags that were sequenced. Because these NILs are derived from a known pedigree of previously genotyped individuals, we were able to filter to include SNPs that would segregate in the progeny, resulting in a final set of 930 loci with high-confidence genotypes for use in subsequent population analyses. A non-trivial fraction of markers remained as missing data in each sample due to the stringent scoring criteria of our method. The majority of uncalled loci in typical $2 \mathrm{~b}-\mathrm{RAD}$ datasets are discarded because of low coverage (Meyer, unpublished observations) so this problem could be mitigated with deeper sequencing. However, the known pedigree of these samples and the low level of recombination made it possible to accurately reconstruct haplotypes despite these missing data. The filtered data were used to construct graphical genotypes [44] of the NIL population, a subset of which are represented in Figure 2. We also provide a database of the genotypes for the entire NIL population [see Additional file 3]. In addition, both parental accessions have been re-sequenced and the genome-wide reads have been deposited in the Short Read Archive (http://www.ncbi.nlm.nih.gov/Traces/sra/ sra.cgi) and posted on the 1001 Genomes Project website (www.1001genomes.org/) so the details of the 930 SNPs utilized in this study can be accessed at these resources.

\section{Genomics of chromosomal introgressions in the NIL population and the added value of increased marker resolution}

Across the 75 NILs, the average number of homozygous introgressions per NIL was 1.35 and ranged from 0 to 4 while the average number of heterozygous introgressions was 2.49 and ranged from 0 to 6 (Figure 3). The average number of introgressions per chromosome was 57.6, ranging from 34 on chromosome 2 to 79 on chromosome 1 [see Additional file 4]. The total length of homozygous introgressions was $506 \mathrm{Mb}$ compared to nearly $949 \mathrm{Mb}$ of heterozygous chromosomal introgression which represent 4.3 and 8.0 times the total length of the Arabidopsis genome, respectively. Together these results suggest we have reached our goal- the entire genome is represented as a Kas-1 introgression for each genotypic state (i.e. zygosity) in at least one NIL, thus enabling QTL validation and fine-mapping for any locus of interest.

The additional loci accounted for by $2 \mathrm{~b}-\mathrm{RAD}$ genotyping resulted in a final marker density of 2.24 markers per cM, based on the estimated $450.8 \mathrm{cM}$ map of the Kas-1 $\times$ Tsu-1 RIL population. This is a significant improvement in resolution from the 0.18 markers per cM when using only the original SSR and Sequenom marker set (hereafter referred to as the coarse map). In spite of the high frequency of uncalled alleles, 128 new introgressions were revealed which summed to nearly $539 \mathrm{Mb}$ of DNA (164 Mb homozygous and $375 \mathrm{Mb}$ heterozygous) that would have been missed without the additional markers from $2 \mathrm{~b}-\mathrm{RAD}$ genotyping. To illustrate this effect we re-sampled the dataset at varying marker densities (Figure 4). The exponential curve fit $\left(r^{2}=0.99\right)$ used to estimate introgression detection begins to level above 800 markers, suggesting diminishing introgression discovery with more extensive genotyping. In a comparison of the NILs using the coarse map relative to the dense map created from 930 2b-RAD markers, the average size of a homozygous introgression in the coarse map was $18 \%$ larger $(1.2 \mathrm{Mb})$ than in the dense map. Similarly, the average heterozygous introgression size in the coarse map was $19 \%$ larger (1.3 Mb), confirming that the additional markers were identifying smaller introgressions missed in the coarse map. This fact is highlighted by the total number of introgressions [see Additional file 4] discovered using the denser marker set. The result was a 1.8-fold (Figure 3) increase in the number of homozygous and heterozygous introgressions discovered.

\section{Case study: utilizing selections from the NIL library for QTL validation and sub-NIL development}

To demonstrate the value of this new resource, we analyzed the RIL population [38] for QTL for night-time leaf conductance $\left(\mathrm{g}_{0}\right) . \mathrm{g}_{0}$ is a low-heritability, quantitative trait that is important for plant-water relations and mineral nutrition. While the adaptive value of $g_{0}$ has yet to be fully understood, incomplete stomatal closure during the night can lead to substantial transpirational water loss [45]. Variation in this trait has been found among and within species, and it correlates with some daytime gas-exchange traits such as water-use efficiency 


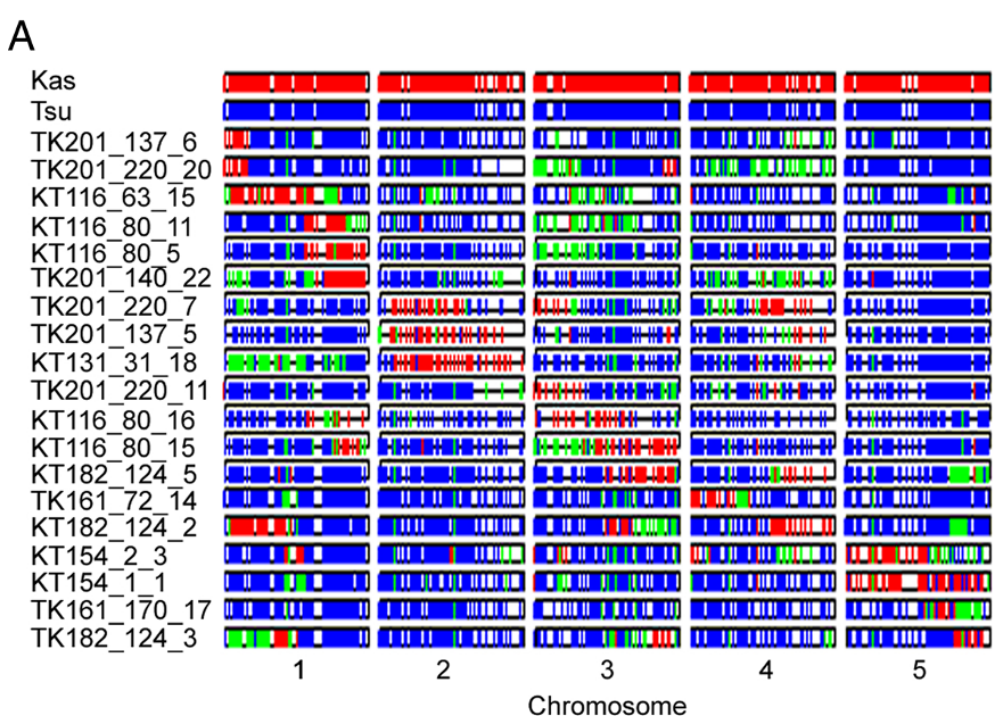

\section{B}

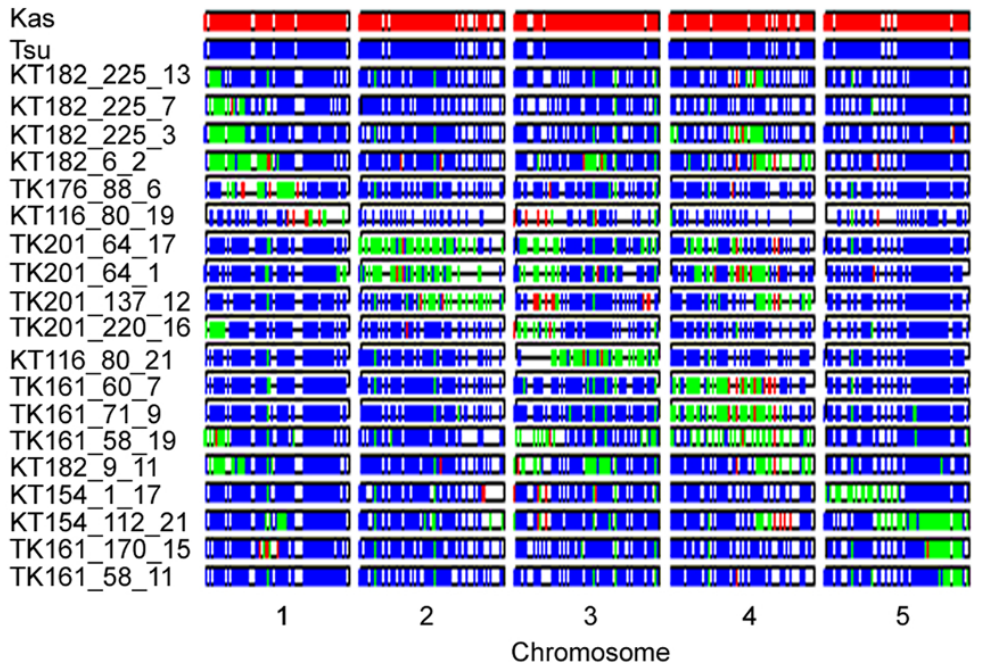

Figure 2 Graphical genotypes of NILs representing (A) homozygous and (B) heterozygous introgressions cumulatively spanning the length of the genome. Red, Kas-1; Blue, Tsu-1; Green, heterozygous.

(the ratio of $\mathrm{CO}_{2}$ assimilation to transpiration) [46]. Estimates of transpiration have been found to be particularly sensitive to $g_{0}$ [47], making it an interesting candidate for studies on the physiology and genetics of plant drought adaptation. In view of that, intraspecific variation in observed $g_{0}$ has been found to have the largest effect on transpiration across a species' native habitat (Bauerle, unpublished observations).

Significant variation in night-time conductance was observed among the RILs. We identified a single QTL for $\mathrm{g}_{0}$ on the top of chromosome 1 (Figure 5A), which explained $9 \%$ of the variance in $g_{0}$, and found the trait to have relatively low broad sense heritability $\left(\mathrm{H}^{2}=\right.$ 0.21 ) in this population. Lines having Kas-1 alleles of markers at the QTL had lower dark conductance, consistent with the dry habitat of the Kas-1 parent [see Additional file 5]. Additional loci were identified on chromosomes 2 and 4 below the threshold of significance, which may have had marginal effects on $g_{0}$ [see Additional file 6].

To validate the QTL we selected two NILs homozygous for a Kas-1 introgression spanning the QTL and measured $g_{0}$ relative to Tsu-1 with the expectation that one or both would have a significantly lower $g_{0}$ value. NIL TK201_137_6 carries an introgression estimated to span physical positions 505,086 to $5,273,972$ on chromosome 1 and KT116_63_15 is estimated to carry a much larger introgression between positions 2,040,091 and 19,225,223 (Figure 5B). KT116_63_15 also carried small heterozygous regions at either end of the homozygous 

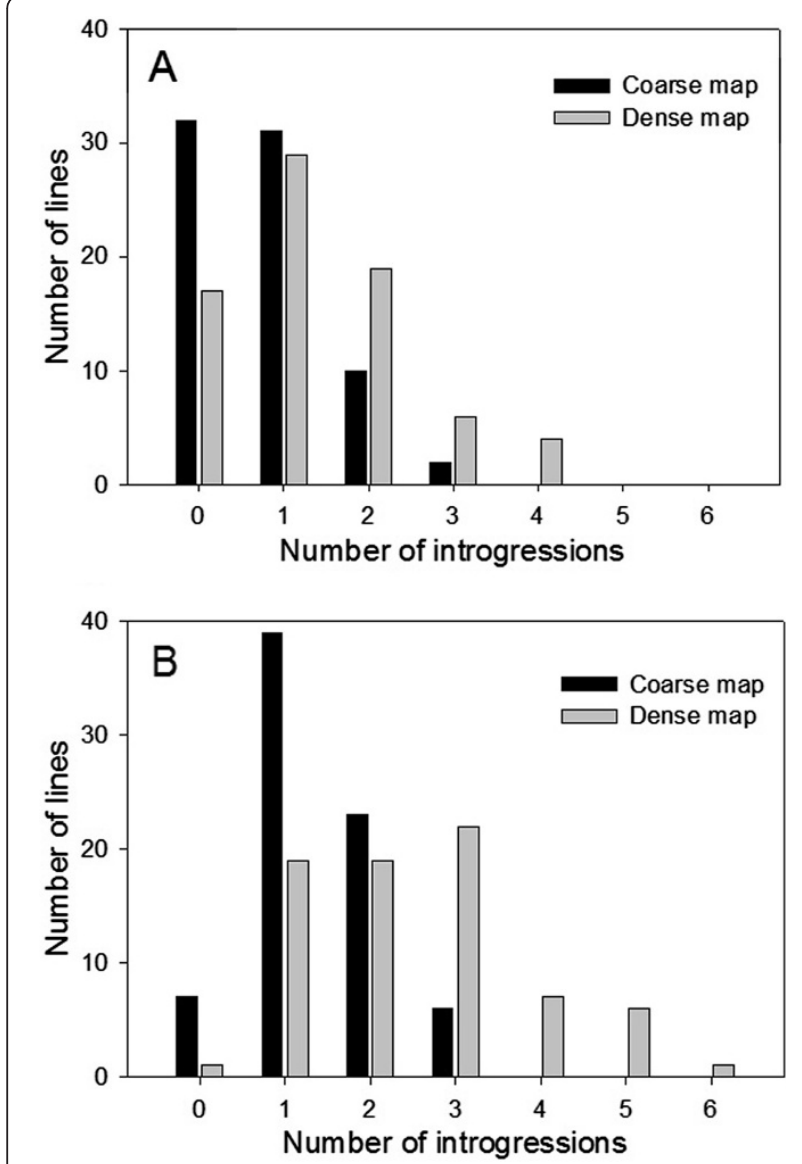

Figure 3 Distribution of (A) homozygous and (B) heterozygous introgression number, estimated using the coarse and dense maps.

introgression. Large and highly significant differences were found between both NILs and Tsu-1 (Table 1), providing strong evidence for the presence of the QTL and providing a surprisingly high estimate of the relative difference in $g_{0}$ conferred by the two alleles when compared to the results of the initial QTL experiment [see Additional file 5]. The region between 5,273,972 and 19,225,223 can be effectively eliminated from consideration for harbouring the causal locus since TK201_ 137_6 was significantly different from Tsu-1 and did not carry Kas-1 DNA in this interval [24]. It is worth noting that both NILs carried introgressions on chromosomes other than the chromosome one focal area. However, none of them were common between the NILs and the difference in $g_{0}$ values between KT116_63_15 and TK201_137_6 was non-significant which suggests these introgressions were not impacting our results substantially.

Nearly 1,500 genes are predicted to lie within the region spanning physical positions 505,086 to 5,273,972 of chromosome one. We have assembled a list of candidate genes based upon hits to gene ontology (GO)

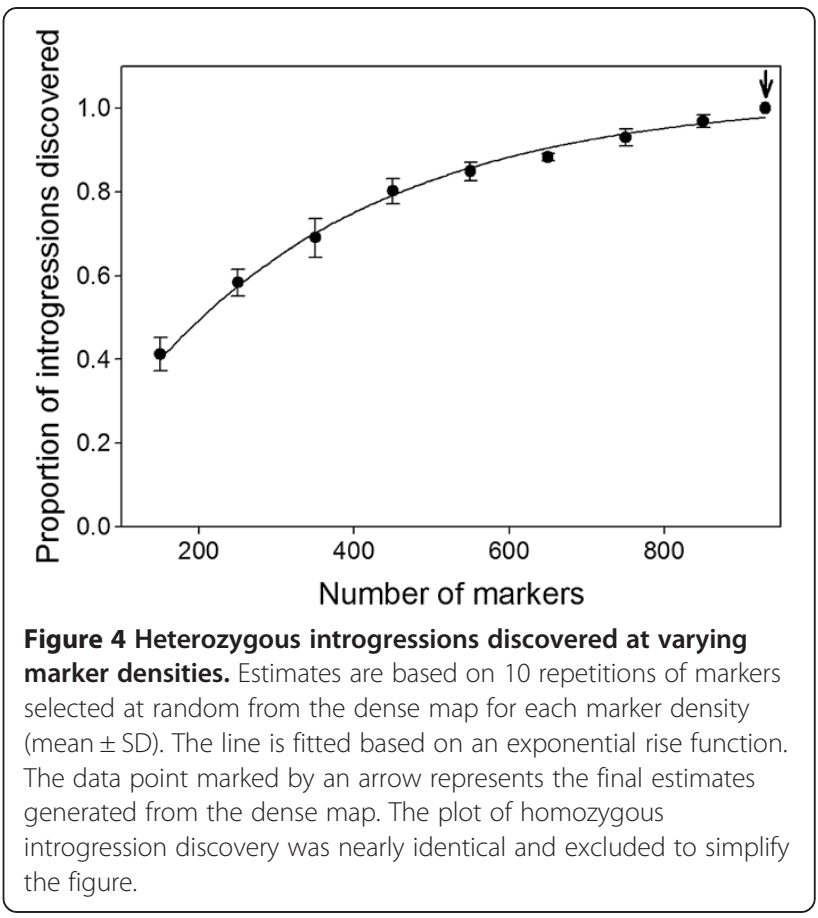

terms relevant to stomatal conductance: abscisic acid (ABA), stomata and water [see Additional file 7].

To illustrate the power of deriving sub-NILs from heterozygote NILs, concurrent to the QTL validation experiment we planted seeds derived from a line heterozygous in the roughly $3 \mathrm{Mb} \mathrm{g}_{0}$ QTL interval (Figure 5). We selected 5 polymorphic loci from a panel of validated SNPs described in [38] for genotyping a population of 286 BC1S3 individuals (BC1S1 graphical genotype is represented in Figure 6). The marker representing the lower end of the interval at physical position 6,839,609 did not segregate and all individuals were homozygous for the Tsu- 1 allele. The genotype for the 2b-RAD allele near this location was scored as "not genotyped" in the original BC1S1 genotyping so we were unsure exactly where this particular heterozygous introgression ended. In the end, we were left with 4 informative markers in the physical interval spanning positions 2,211,035 to $6,572,582$. We selected 17 recombinants (Figure 6) representing the majority of the recombination events possible. Unfortunately, no double recombinants were discovered so that a sub-NIL representing the Kas-1 alleles at the middle of the interval could be recovered. However, heterozygous individuals TK176_108_1_4_13 and TK176_108_1_4_38 were kept for selfing and will be available for re-planting to accomplish this since a crossover has already occurred at the lower end. Ultimately, individuals were recovered in this single selfing generation that could be used in the next generation for $g_{0}$ phenotyping experiments to effectively narrow the QTL interval down to, at most, the $1.9 \mathrm{Mb}$ interval between 


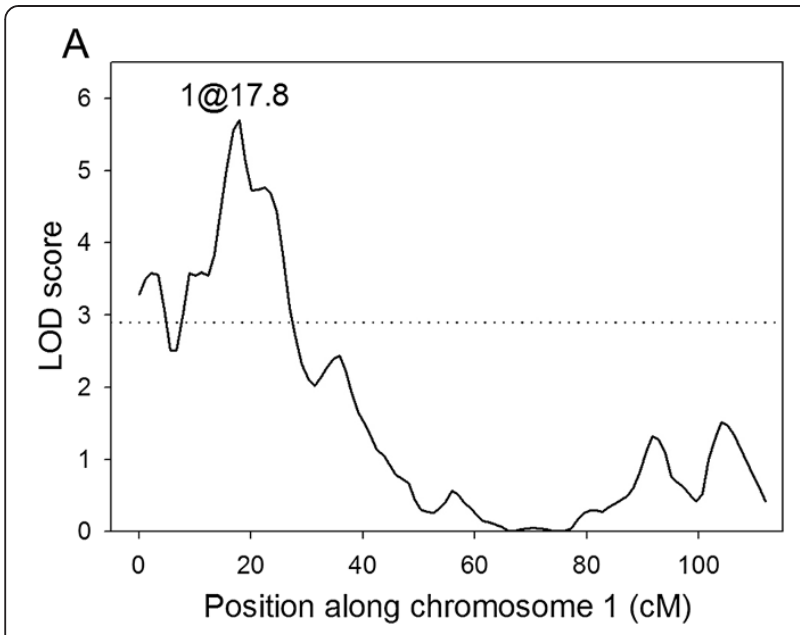

\section{B}

TK201_137_6

KT116_63_15

Figure 5 QTL location and graphical genotypes of the NILs used in the QTL validation. (A) Localization of the darkconductance QTL along chromosome 1, the dotted line indicates the threshold LOD score. (B) Graphical genotypes scaled to represent the genetic distance (CM) of the $\mathrm{x}$-axis of panel $\mathrm{A}$ of chromosome 1 for the NILs used in the QTL validation.

markers C1_2211035 and C1_4142402, an interval predicted to carry about 590 genes.

\section{Discussion}

Maintenance of homozygous and heterozygous NILs facilitates simultaneous QTL validation and fine-mapping efforts

Near-isogenic lines remain the ideal starting material for validation of QTL as well as breeding schemes designed for fine-mapping with the end goal being the identification of candidate genes [48-51]. QTL validation is relatively straightforward and consists simply of phenotyping NILs with introgressions at the region of interest for the trait of interest. Creation of a suitable population for fine mapping is not as straightforward and is normally a three-generation process that starts with a cross between an inbred NIL and the recurrent parent. This is typically

Table 1 Results of QTL validation experiment comparing NIL go values with Tsu-1

\begin{tabular}{ccccc}
\hline Comparison & $\begin{array}{c}\mathbf{g}_{\mathbf{0}} \\
(\mathbf{m m o l} \mathbf{~ m - 2} \mathbf{~ s - 1 )}\end{array}$ & $\begin{array}{c}\text { Standard } \\
\text { Error }\end{array}$ & $\begin{array}{c}\text { Difference } \\
\text { NIL - (Tsu-1) }\end{array}$ & t value \\
\hline Tsu-1 & 119.76 & 11.55 & $\mathrm{n} / \mathrm{a}$ & -0.94 \\
TK201_137_6 & 52.42 & 10.99 & $-67.34^{* *}$ & -4.02 \\
KT116_63_15 & 67.45 & 11.40 & $-52.30^{* *}$ & -3.15 \\
\hline
\end{tabular}

Difference values with ${ }^{* *}$ are highly significant $(P<0.0001)$. followed by a generation of self-pollination to allow for recombination in the introgression region. The seed harvested from these self-pollinated plants can then be genotyped with markers specific to the region so that homozygous sub-NILs can be identified. The process is fairly straightforward and inexpensive in the context of physical resource, but there is a time cost of at least 3 generations (equivalent to a minimum of 18 weeks).

Our case study illustrates the advantages of maintaining both homozygotes and heterozygotes in the NIL population, combining the benefits of traditional homozygous NILs with the advantages of HIFs [22,23]. For example, measuring $g_{0}$ on the homozygous NILs provided strong evidence for the presence of the QTL in a single generation, thus avoiding the process of generating homozygous lines that would be necessary in HIF populations. These results provided a better estimate of the QTL effect size relative to the results derived from our QTL mapping approach and have justified further investments in finemapping using heterozygous NILs. This emphasizes the power NILs create by isolating the genetic factors controlling a phenotype to a single locus as there were other loci worthy of consideration as contributors to variation in $\mathrm{g}_{0}$ in the RIL population. Analysis of the genes predicted to lie within this interval revealed a majority of them had GO annotations related to ABA, the major signalling molecule in stomatal regulation [52-54], but examination of the entire region with the AmiGO enrichment analysis tool [55] found it was not significantly enriched for ABA genes. Inspection of the physical location of these ABA-associated candidates reveals that they are clustered in a $1.2 \mathrm{Mb}$ interval (At 1 physical interval: 712,473-1,894,148) which represents a relatively small portion of the $4.8 \mathrm{Mb}$ introgression tested, thus providing an interesting focal region during fine-mapping of the $g_{0}$ phenotype.

With regards to fine-mapping, selfing a heterozygous NIL selection from the population yielded several subNILs suitable for phenotyping or additional genotyping in future generations, an attribute common with HIF populations and advantageous over traditional NILs. This was accomplished using a modest population size of BC1S3 plants $(n=286)$ and the interval could be narrowed down further through genotyping at a higher number of loci and increasing the population size [56]. Regardless, in a six-week period we have identified a population encompassing recombinants in the $4.8 \mathrm{Mb}$ region identified as causal during the QTL validation experiment, translating to a 3 -fold change in total time versus a breeding scheme utilizing inbred NILs.

\section{2b-RAD is an efficient method for dense genotyping of recombinant populations \\ Arabidopsis thaliana recently celebrated its 25th anni-} versary as a model organism and now stands alone as 


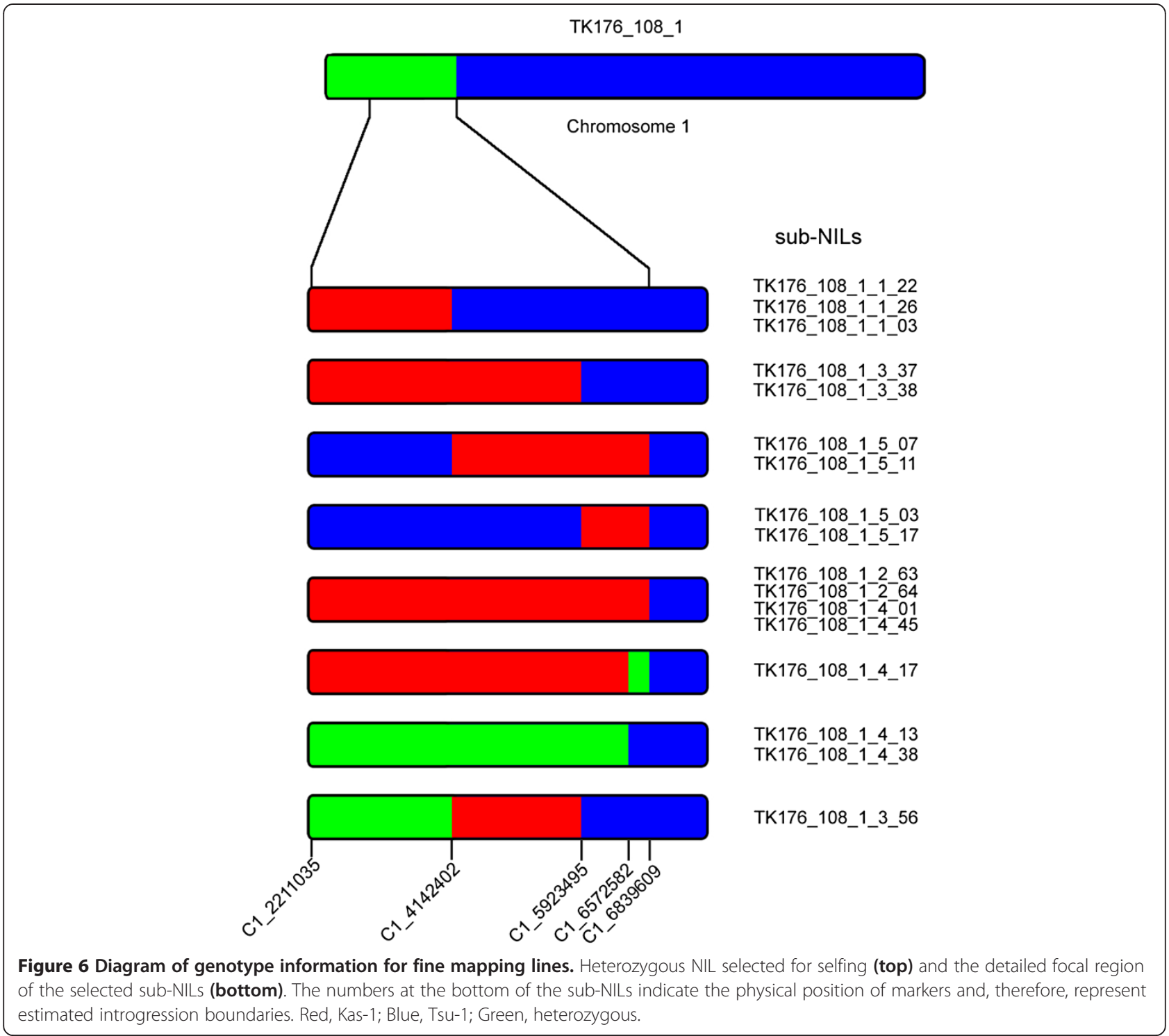

the most thoroughly studied plant species on record http://www.arabidopsis.org/, [57]. Recent efforts are producing comprehensive polymorphism databases (http:// www.arabidopsis.org/, http://signal.salk.edu/cgi-bin/AtSFP). To interpret the significance and functional consequences of this natural variation, we need to understand the multivariate phenotypic consequences of these variants. NIL libraries, mutants and complementation studies are the tools required for this mechanistic understanding.

The 2b-RAD method added an additional 930 high confidence genotypes to our map providing a level of resolution not yet achieved in any of the Arabidopsis NIL populations described to date. The value of these additional markers is obvious as we compare the coarse and dense maps. The discovery of an additional 129 introgressions is clearly important when making selections for QTL validation. For instance, three additional homozygous introgressions were discovered in KT154_2_3, changing the estimate from one to four. This is a clear illustration of the risks associated with utilizing NILs genotyped at low density in experiments aimed at QTL validation. These offsite introgressions may have effects on the phenotype of interest, potentially resulting in erroneous or uncertain conclusions regarding the QTL effect size and location.

The Kas- $1 \times$ Tsu- 1 RIL and NIL populations are a valuable resource for research on the genetics of drought adaptation in the Brassicaceae

Substantial variation for several traits relevant to drought adaptation have been observed in the Kas- $1 \times$ Tsu- 1 RIL population including $\delta^{13} \mathrm{C}$, leaf water content, 
instantaneous transpiration rate, flowering time, abscisic acid content and root mass [38], unpublished results]. Accordingly, the NIL population described herein is expected to vary for the same traits, providing a powerful resource for moving from QTL, encompassing thousands of genes, discovered in the RIL population towards a smaller list of putative functional candidates.

No other plant species has been more studied or characterized than Arabidopsis thaliana [57]. A high degree of sequence collinearity between it and members of the agriculturally significant Brassica genus was discovered over a decade ago [58]. Similar levels of synteny have been found in comparisons with other taxa in the Brassicaceae [59-62]. These results suggest that translational genomics, that is utilizing basic research findings in model organisms to answer practical research questions in species of higher economic value or importance $[63,64]$, could be a viable avenue in understanding complex traits. In this regard, we suggest the Kas- $1 \times$ Tsu- 1 populations as the ideal starting point for basic research on the genetics and genomics of drought adaptation.

\section{Conclusions}

We have developed a population of 75 NILs that provides genetic resources for fine-mapping QTL as well as QTL corroboration. The high marker density used to construct the population provides a level of resolution not yet seen in a NIL population, thus minimizing ambiguity in fine-mapping and QTL validation studies caused by unidentified chromosomal introgressions elsewhere in the genome. The unique variation that exists between the parents used to construct this resource provides a valuable asset for research focused on identifying the genes responsible for drought adaptation in Arabidopsis and beyond.

\section{Methods}

\section{Plant material \& growth conditions}

The A. thaliana accessions Kas-1 (CS903) and Tsu-1 (CS1640) were used as the original parent lines in developing the RIL population of 346 lines. Kas-1 and Tsu-1 were chosen as parents for developing this population as a result of their extreme differences in water use efficiency as measured by $\delta^{13} \mathrm{C}[39,40]$. RILs from this population served as the starting point for the NIL breeding program described below.

For the QTL experiment, seed of the RILs along with the parents were sown on soil (Fafard 4P mix, Conrad Fafard Inc., Agawam, MA) in 3-inch pots. Seeds were planted in a randomized complete block design consisting of 2 blocks, and then the pots were refrigerated at $4^{\circ} \mathrm{C}$ in darkness for $5 \mathrm{~d}$ to cold-stratify the seeds prior to commencement of a 8:16 h (light: dark) photoperiod in Conviron ATC60 growth chambers (Controlled
Environments, Winnipeg, $\mathrm{MB}$ ), at $23^{\circ} \mathrm{C}$ and $40 \%$ humidity during the day and $20^{\circ} \mathrm{C}$ and $50 \%$ humidity during the dark period. Light intensity was approximately $330 \mu \mathrm{mol} \mathrm{m}^{-2} \mathrm{~s}^{-1}$. Plants were grown for approximately 6 weeks prior to measurement. Stomatal conductance was measured in darkness on non-senescing leaves that were large enough to fully accommodate the leaf chamber $(1 \mathrm{~cm} \times 2 \mathrm{~cm})$, using an infrared gas analyser (model Li-Cor 6400, LiCor Inc., Lincoln, NE). Prior to measurement the plants were dark adapted for $20-28 \mathrm{~h}$. A humidifier was used to reduce variation in humidity over the course of the measurements. For each leaf 10 measurements were taken, with an interval of $10 \mathrm{~s}$ between measurements.

For the QTL validation experiment, plants were grown in a randomized complete block design consisting of 3 blocks where each genotype was replicated 6 times within each block. Plants were grown under exactly the same conditions as those described above except that the photoperiod was increased to $12 ; 12 \mathrm{~h}$ (light:dark) to accommodate other experiments conducted in the same chamber. One major difference between the two experiments was the use of leaf porometers (model SC-1, Decagon Devices, Inc., Pullman, WA) rather than an infrared gas analyser for stomatal conductance estimates. Two non-senescing leaves were measured on each plant following the manufacturer's recommended protocol.

\section{Genetic analyses}

Broad-sense heritability was estimated by calculating the ratio $V_{G}: V_{P}$, where $V_{G}$ is the among-RIL component of variance and $V_{P}$ is the total phenotypic variance. QTL mapping was performed in the $\mathrm{R} / \mathrm{qtl}$ program of the $\mathrm{R}$ statistical package $[65,66]$, using Haley-Knott regression. Significance thresholds were determined using 1000 permutations. A penalized stepwise approach [67] was used for selection of a multiple-QTL model.

For the QTL validation experiment, data were analyzed with a linear mixed model using PROC MIXED in the SAS software package (SAS Institute Inc. 2003, Cary, NC) where block, row and column effects were treated as random.

\section{Marker assisted NIL breeding program}

To start, 7 RILs were selected from the original population of 346 using the code supplied in an additional file [see Additional file 1]. These 7 represented lines homozygous for Kas- 1 alleles across one of each of the 5 chromosomes and all were crossed to Tsu-1 at least 10 times. Some attempted crosses may result in selfpollination due to technical error, thus we genotyped progeny to confirm they were F1s. In general, the real F1s were several times larger than the midparent value, so genotyping was almost unnecessary. Confirmed F1s were crossed back to Tsu-1 and each fruit was collected 
separately and considered a BC1 family, ultimately creating 25 families. 24 plants from each family were genotyped at the chromosome of interest and selected for selfing to generate $\mathrm{BC} 1 \mathrm{~S} 1$ seed. In addition to culling the occasional plant generated due to self-pollination, it was also necessary to remove individuals sired by (haploid) pollen from the F1 carrying Tsu-1 alleles for the chromosome of interest. In the next generation, 690 BC1S1 plants were genotyped with the 48 genome-wide SSRs described in [38]. These were then ranked using an algorithm [see Additional file 2] to find lines that were largely Tsu-1, but carrying Kas-1 introgressions spanning the genome. In the end, 75 lines were selected which we screened at an additional 149 loci using the Sequenom MassARRAY ${ }^{\oplus}$ platform, of which 41 were polymorphic. 930 polymorphic loci were added to this marker data set via $2 \mathrm{~b}-\mathrm{RAD}$ [41] where class IIB restriction enzymes are used minimize genome complexity for a final total of 1011 genotyped.

\section{DNA extraction and genotyping}

Genomic DNA was isolated from lyophilized tissue collected from approximately 4-week-old, chamber grown plants using the DNeasy Plant Mini Kit (Qiagen, Valencia, CA) according to the manufacturer's instructions.

The 48 polymorphic microsatellites used in this study were selected from the large number of those available in A. thaliana [68,69], arabidopsis.org] due to easily distinguishable allele calls. Descriptions of the primers, PCR conditions and allele scoring are explained in [38].

DNA samples were used to prepare 2b-RAD libraries as previously described [41]. A detailed protocol is available at the Meyer laboratory website (http://people. oregonstate.edu/ meyere/). Briefly, library preparation for $2 \mathrm{~b}-\mathrm{RAD}$ genotyping began with digestion of gDNA samples with AlfI (Fermentas) for $37^{\circ} \mathrm{C}$ for $3 \mathrm{~h}$ followed by ligation of adaptors at $4^{\circ} \mathrm{C}$ for $16 \mathrm{~h}$. Ligation products were amplified by PCR and barcodes introduced to gelextracted products in a second PCR reaction. Finally, libraries were pooled for multiplex sequencing on the SOLiD sequencing platform (Applied Biosystems). Raw sequences were processed to exclude low-quality reads, and the HQ reads that remained aligned in color-space using the SHRiMP software package [70] to AlfI sites extracted from the Arabidopsis genome (TAIR9). A custom Perl script was applied to eliminate short, statistically weak and ambiguous alignments (reads matching multiple sites equally well). Finally, genotypes were determined from nucleotide frequencies using custom Perl scripts to classify each locus as homozygous (minor allele frequencies [MAF] <1\%), heterozygous (MAF $>25 \%$ ), or undetermined ( $1 \%>$ MAF $>25 \%)$. 20× coverage was required in the parental genomes to identify these alleles with high confidence, and a relaxed threshold of $10 \times$ in all other samples to maximize marker densities. Each polymorphic locus identified in these genotypes was compared with the parental genotypes (Tsu-1 and Kas-1) to assign it to one of these backgrounds, a comparison that would obviously not be possible for any loci genotyped in one parent but not the other as a result of variation in sequencing coverage. To reduce the effects of such missing data, we imported genotypes for Tsu-1 and Kas-1 from resequencing data (McKay, unpublished results) for any loci genotyped in one parent but not the other.

KASP SNP genotyping assays (LGC Genomics, Teddington, Middlesex, UK) were used for sub-NIL development. Primer sequences [see Additional file 8] were designed using sequence data from TAIR10 [71] for amplification of SNPs identified and validated on the SNPlex genotyping system (Applied Biosystems) as described in [38]. KASP is a novel allele-specific PCR assay that utilizes a FRET (Fluorescence Resonance Energy Transfer) system. In short, along with a common primer, allele-specific primers are designed to include a unique $18 \mathrm{bp}$ sequence at the $5^{\prime}$ end. The unique sequences are identical to a pair of oligonucleotides with 3' bound quenchers for a complement pair of 5 ' fluorescently labelled oligos inside the reaction mix. During PCR, allele specific amplification leads to the generated product(s) outcompeting the quencher containing oligos for binding to the fluorescently labelled oligos, allowing for an observable signal to be measured using a light reader. The intensity of the signal(s) allows for a quantitative measure of SNP copy number.

\section{Estimating chromosomal introgression length and number}

The physical length of introgressions in the final NIL library was estimated using graphical genotypes [44]. Physical length estimates of introgressions flanked by SSR markers were made using the location of the forward primers, SNP locations were determined by their location in the Col-0 reference genome. To avoid false-positives, an introgression was scored based on the presence of at least 3 consecutive markers with the Kas-1 genotype. Introgression boundaries were then defined by three consecutive markers with an alternative genotype. This helped avoid over-estimating introgression numbers due to occasional incorrect allele calls or differences in the location of loci in this population relative to the Col-0 genome used as a reference for mapping sequence reads. For the analysis of introgression discovery at varying marker densities an Excel Macro was written to sum the number of heterozygous and homozygous introgressions discovered. The loci included in replicated sampling were selected randomly using Excel's RAND function. 


\section{Candidate gene identification}

The full list of genes expected to lie within the QTL interval spanning physical positions 505,086 to 5,273,972 was assembled using TAIR10 [71]. GO annotations for the full gene list were downloaded using the Bulk Data Retrieval and Analysis tool on TAIR10 and searched using the terms abscisic acid (ABA), stomata and water. Gene enrichment analysis was performed using the GO enrichment analysis tool in AmiGO [55].

\section{Additional files}

\section{Additional file 1: $\mathrm{R}$ script used to select RILs for backcrossing. \\ Additional file 2: R script used to select NILs for introgressions spanning the genome.}

Additional file 3: CSV Table of genotypes of the NIL population. Additional file 4: PDF Table summarizing the number introgressions discovered on each chromosome in the coarse and dense maps.

Additional file 5: TIF Bar graph of mean dark conductance of RILs carrying the Kas-1 allele at the QTL on chromosome 1 relative to RILs carrying the Tsu- 1 allele.

Additional file 6: TIF Genome-wide LOD graph of $g_{0}$ QTL scans. Additional file 7: CSV Table of candidate genes in QTL interval selected based on relevant GO terms.

Additional file 8: CSV Table of the primer sequences used for KASP assays.

\section{Competing interests}

The authors declare that they have no competing interests.

\section{Authors' contributions}

SS, TEJ and JKM conceived the study. SY organized and conducted the backcrossing and selfing. SS wrote and implemented the algorithms for selecting RILs and backcross lines. EM produced the sequencing libraries and analyzed the genotype data with RSF. GR, WLB, JLM and JKM performed the stomatal conductance phenotyping experiments. RSF and JLM analyzed the quantitative genetic data. RSF wrote the manuscript with JLM and JKM. All authors read and approved the final manuscript.

\section{Acknowledgements}

This research was supported by NSF grants DEB-1022196 and DEB-0618302 to JKM and DEB-0618347 and IOS-09221457 to TEJ.

\section{Author details}

'Department of Bioagricultural Sciences \& Pest Management, Colorado State University, 80523 Fort Collins, CO, USA. ${ }^{2}$ Cargill Specialty Seeds \& Oils, 80525 Fort Collins, CO, USA. ${ }^{3}$ Department of Horticulture and Landscape Architecture, Colorado State University, 80523 Fort Collins, CO, USA. ${ }^{4}$ Department of Epidemiology and Biostatistics, University of California San Francisco, 94143 San Francisco, CA, USA. ${ }^{5}$ Department of Zoology, Oregon State University Corvallis, 97331 Corvallis, OR, USA. ${ }^{6}$ Section of Integrative Biology \& Institute of Cellular and Molecular Biology, University of Texas, 78712 Austin, TX, USA.

Received: 11 March 2013 Accepted: 20 September 2013 Published: 25 September 2013

\section{References}

1. Sax K: The association of size differences with seed-coat pattern and pigmentation in Phaseolus vulgaris. Genetics 1923, 8:552-560.

2. Soller M, Brody T: On the power of experimental designs for the detection of linkage between marker loci and quantitative loci in crosses between inbred lines. Theor Appl Genet 1976, 47:35-39.
3. Lander ES, Botstein D: Mapping Mendelian factors underlying quantitative traits using RFLP linkage maps. Genetics 1989, 121:185-199.

4. Haley CS, Knott SA: A simple regression method for mapping quantitative trait loci in line crosses using flanking markers. Heredity 1992, 69:315-324

5. Jansen RC: Interval mapping of multiple quantitative trait loci. Genetics 1993, 1:205-211.

6. Zeng ZB: Theoretical basis for separation of multiple linked gene effects in mapping quantitative trait loci. Proc Natl Acad Sci USA 1993, 23:10972-10976.

7. Zeng ZB: Precision mapping of quantitative trait loci. Genetics 1994, 4:1457-1468.

8. Sen S, Churchill GA: A statistical framework for quantitative trait mapping. Genetics 2001, 159:371-387.

9. Price AH: Believe it or not, QTLs are accurate! Trends Plant Sci 2006 11:213-216.

10. Rockman MV: Reverse engineering the genotype-phenotype map with natural genetic variation. Nature 2008, 456:738-744.

11. Monforte AJ, Tanksley SD: Development of a set of near isogenic and backcross recombinant inbred lines containing most of the Lycopersicon hirsutum genome in a L. esculentum genetic background: a tool for gene mapping and gene discovery. Genome 2000, 43:803-813.

12. Landi P, Sanguineti MC, Salvi S, Giuliani S, Bellotti M, Maccaferri M, Conti S, Tuberosa R: Validation and characterization of a major QTL affecting leaf ABA concentration in maize. Mol Breeding 2005, 15:291-303.

13. Iniguez-Luy FL, Lukens L, Farnham MW, Amasino RM, Osborn TC: Development of public immortal mapping populations, molecular markers and linkage maps for rapid cycling Brassica rapa and B. Oleracea. Theor Appl Genet 2009, 120:31-43.

14. Eshed Y, Zamir D: A genomic library of Lycopersicon pennellii in L. esculentum: a tool for fine mapping of genes. Euphytica 1994, 79:175-179.

15. Frary A, Nesbitt TC, Grandillo S, van der Knaap E, Cong B, Liu JP, Meller J, Elber R, Alpert KB, Tanksley SD: fw2.2: a quantitative trait locus key to the evolution of tomato fruit size. Science 2000, 289:85-88.

16. Takahashi Y, Shomura A, Sasaki T, Yano M: Hd6, a rice quantitative trait locus involved in photoperiod sensitivity, encodes the alpha subunit of protein kinase CK2. Proc Natl Acad Sci USA 2001, 98:7922-7927.

17. Lin $\mathrm{H}$, Liang ZW, Sasaki T, Yano M: Fine mapping and characterization of quantitative trait loci $\mathrm{Hd} 4$ and $\mathrm{Hd} 5$ controlling heading date in rice. Breeding Sci 2003, 53:51-59.

18. Juenger TE, Wayne T, Boles S, Symonds W, McKay J, Coughlan SJ: Natural genetic variation in whole-genome expression in Arabidopsis thaliana: the impact of physiological QTL introgression. Mol Ecol 2005, 15:1351-1365.

19. Gerald JNF, Lehti-Shiu MD, Ingram PA, Deak KI, Biesiada T, Malamy JE: Identification of quantitative trait loci that regulate Arabidopsis root system size and plasticity. Genetics 2006, 172:485-498.

20. Xing YZ, Tang WJ, Xue WY, Xu CG, Zhang Q: Fine mapping of a major quantitative trait loci, qSSP7, controlling the number of spikelets per panicle as a single Mendelian factor in rice. Theor Appl Genet 2008, 116:789-796.

21. Zhou L, Zeng Y, Zheng W, Tang B, Yang S, Zhang H, Li J, Li Z: Fine mapping a QTL qCTB7 for cold tolerance at the booting stage on rice chromosome 7 using a near-isogenic line. Theor Appl Genet 2010, 121:895-905.

22. Tuinstra MR, Ejeta G, Goldsbrough PB: Heterogeneous inbred family (HIF) analysis: a method for developing near-isogenic lines that differ at quantitative trait loci. Theor Appl Genet 1997, 95:1005-1011.

23. Loudet O, Gaudon V, Trubuil A, Daniel-Vedele F: Quantitative trait loci controlling root growth and architecture in Arabidopsis thaliana confirmed by heterogeneous inbred family. Theor Appl Genet 2005, 110:742-753.

24. Eshed Y, Zamir D: An introgression line population of Lycopersicon pennellii in the cultivated tomato enables the identification and fine mapping of yield-associated QTL. Genetics 1995, 141:1147-1162.

25. Monforte AJ, Tanksley SD: Fine mapping of a quantitative trait locus (QTL) from Lycopersicon hirsutum chromosome 1 affecting fruit characteristics and agronomic traits: breaking linkage among QTLs affecting different traits and dissection of heterosis for yield. Theor Appl Genet 2000, 100:471-479. 
26. Koumproglou R, Wilkes TM, Townson P, Wang XY, Beynon J, Pooni HS, Newbury HJ, Kearsey MJ: STAIRS: a new genetic resource for functional genomic studies of Arabidopsis. Plant J 2002, 31:355-364.

27. Keurentjes JJB, Bentsink L, Alonso-Blanco C, Hanhart CJ, De Blankestijn Vries H, Effgen S, Vreugdenhil D, Koornneef M: Development of a near-isogenic line population of Arabidopsis thaliana and comparison of mapping power with a recombinant inbred line population. Genetics 2007, 175:891-905.

28. Alonso-Blanco C, Peeters AJM, Koornneef M, Lister C, Dean C, van den Bosch N, Pot J, Kuiper MTR: Development of an AFLP based linkage map of Ler, Col and Cvi Arabidopsis thaliana ecotypes and construction of a Ler/Cvi recombinant inbred line population. Plant J 1998, 14:259-271.

29. Juenger TE, Mckay JK, Hausmann N, Keurentjes JJB, Sen S, Stowe KA, Dawson TE, Simms EL, Richards JH: Identification and characterization of QTL underlying whole-plant physiology in Arabidopsis thaliana: $813 C$, stomatal conductance and transpiration efficiency. Plant Cell Environ 2005, 28:697-708.

30. Esch E, Szymaniak JM, Yates H, Pawlowski WP, Buckler ES: Using crossover breakpoints in recombinant inbred lines to identify quantitative trait loci controlling the global recombination frequency. Genetics 2007, 177:1851-1858.

31. Laserna MP, Sanchez RA, Botto JF: Light-related Loci Controlling Seed Germination in Ler $\times$ Cvi and Bay $-0 \times$ Sha Recombinant Inbred-line Populations of Arabidopsis thaliana. Ann Bot-London 2008, 102:631-642.

32. Waters BM, Grusak MA: Quantitative trait locus mapping for seed mineral concentrations in two Arabidopsis thaliana recombinant inbred populations. New Phytol 2008, 179:1033-1047.

33. Li P, Wind JJ, Shi X, Zhang H, Hanson J, Smeekens SC, Teng S: Fructose sensitivity is suppressed in Arabidopsis by the transcription factor ANAC089 lacking the membrane-bound domain. Proc Natl Acad Sci USA 2011, 108:3436-3441

34. Torjek O, Meyer RC, Zehnsdorf M, Teltow M, Strompen G, Witucka-Wall H, Blacha A, Altmann T: Construction and analysis of 2 reciprocal introgression line populations. J Hered 2008, 99:396-406.

35. Torjek O, Berger D, Meyer RC, Mussig C, Schmid K, Sorensen TR, Weisshaar B, Mitchell-Olds T, Altmann T: Establishment of a high-efficiency SNPbased framework marker set for Arabidopsis. Plant J 2003, 36:122-140.

36. Reif JC, Kusterer B, Piepho HP, Meyer RC, Altmann T, Schon CC, Melchinger AE: Unraveling epistasis with triple testcross progenies of near-isogenic lines. Genetics 2009, 181:247-257.

37. Jan Lisec J, Steinfath M, Meyer RC, Selbig J, Melchinger AE, Willmitzer L, Altmann T: Identification of heterotic metabolite QTL in Arabidopsis thaliana RIL and IL populations. Plant J 2009, 59:777-788.

38. McKay JK, Richards JH, Nemali KS, Sen S, Mitchell-Olds T, Boles S, Stahl EA, Wayne T, Juenger TE: Genetics of drought adaptation in Arabidopsis thaliana II. QTL analysis of a new mapping population, Kas- $1 \times$ Tsu-1. Evolution 2008, 62-12:3014-3026.

39. McKay JK, Richards JH, Mitchell-Olds T: Genetics of drought adaptation in Arabidopsis thaliana: I. Pleiotropy contributes to genetic correlations among ecological traits. Mol Ecol 2003, 12:1137-1151.

40. Juenger TE, Sen S, Bray E, Stahl E, Wayne T, McKay J, Richards JH: Exploring genetic and expression differences between physiologically extreme ecotypes: comparative genomic hybridization and gene expression studies of Kas-1 and Tsu-1 accessions of Arabidopsis thaliana. Plant Cell Environ 2010, 33:1268-1284.

41. Wang S, Meyer E, McKay JK, Matz MV: 2b-RAD: a simple and flexible method for genome-wide genotyping. Nat Methods 2012, 9:808-810

42. Davey JW, Hohenlohe PA, Etter PD, Boone JQ, Catchen JM, Blaxter ML: Genome-wide genetic marker discovery and genotyping using next-generation sequencing. Nat Rev Genet 2011, 12:499-510.

43. Clark AG, Hubisz MJ, Bustamante CD, Williamson SH, Nielsen R: Ascertainment bias in studies of human genome-wide polymorphism. Genome Res 2005, 15:1496-1502.

44. Young ND, Tanksley SD: Restriction fragment length polymorphism maps and the concept of graphical genotypes. Theor App/ Genet 1989, 77:95-101.

45. Caird MA, Richards JH, Donovan LA: Nighttime stomatal conductance and transpiration in $C_{3}$ and $C_{4}$ plants. Plant Physiol 2007, 143:4-10.

46. Christman MA, Richards JH, McKay JK, Stahl EA, Juenger TE, Donovan LA Genetic variation in Arabidopsis thaliana for night-time leaf conductance. Plant Cell Environ 2008, 31:1170-1178.
47. Bauerle WL, Bowden JD: Separating foliar physiology from morphology reveals the relative roles of vertically structured transpiration factors within red maple crowns and limitations of larger scale models. J Exp Bot 2011, 62:4295-4307.

48. Yan WH, Wang $P$, Chen HX, Zhou HJ, Li QP, Wang CR, Ding ZH, Zhang YS, Yu SB, Xing YZ, Zhang QF: A major QTL, Ghd8, plays pleiotropic roles in regulating grain productivity, plant height, and heading date in rice. $\mathrm{Mol}$ Plant 2011, 4:319-330.

49. Wang S, Wu K, Yuan Q, Liu X, Liu Z, Lin X, Zeng R, Zhu H, Dong G, Qian Q, Zhang G, Fu X: Control of grain size, shape and quality by OSSPL16 in rice. Nat Genet 2012, 44:950-955.

50. Paterson AH, DeVerna JW, Lanini B, Tanksley SD: Fine mapping of quantitative trait loci using selected overlapping recombinant chromosomes, in an interspecies cross of tomato. Genetics 1990, 124:735-742.

51. Ducrocq S, Giauffret C, Madur D, Combes V, Dumas F, Jouanne S, Coubriche $D$, Jamin P, Moreau L, Charcosset A: Fine mapping and haplotype structure analysis of a major flowering time quantitative trait locus on maize chromosome 10. Genetics 2009, 183:1555-1563.

52. Acharya BR, Assmann SM: Hormone interactions in stomatal function. Plant Mol Biol 2009, 69:451-462.

53. Nilson SE, Assmann SM: The control of transpiration. Insights from Arabidopsis. Plant Physiol 2007, 143:19-27.

54. Schroeder Jl, Allen GJ, Hugouvieux V, Kwak JM, Waner D: Guard cell signal transduction. Annu Rev Plant Physiol Plant Mol Biol 2001, 52:627-658.

55. Carbon S, Ireland A, Mungall CJ, Shu S, Marshall B, Lewis S: the AmiGO Hub, the Web Presence Working Group: AmiGO: online access to ontology and annotation data. http://bioinformatics.oxfordjournals.org/cgi/content/ abstract/25/2/288

56. Dinka SJ, Campbell MA, Demers T, Raizada MN: Predicting the size of the progeny mapping population required to positionally clone a gene. Genetics 2007, 176:2035-2054.

57. Koornneef M, Meinke D: The development of Arabidopsis as a model plant. Plant J 2010, 61:909-921

58. Cavell AC, Lydiate DJ, Parkin IAP, Dean C, Trick M: Collinearity between a 30-centimorgan segment of Arabidopsis thaliana chromosome 4 and duplicated regions within the Brassica napus genome. Genome 1998, 41:62-69.

59. Boivin K, Acarkan A, Mbulu R, Clarenz O, Schmidt R: The Arabidopsis genome sequence as a tool for genome sequence analysis in Brassicaceae. A comparison of the Arabidopsis and Capsella rubella genomes. Plant Physiol 2004, 135:735-744.

60. Yogeeswaran K, Frary A, York TL, Amenta A, Lesser AH, Nasrallah JB, Tanksley SD, Nasrallah ME: Comparative genome analyses of Arabidopsis spp.: Inferring chromosomal rearrangement events in the evolutionary history of $A$. thaliana. Genome Res 2005, 15:505-515.

61. Lysak MA, Berr A, Pecinka A, Schmidt R, McBreen K, Schubert I: Mechanisms of chromosome number reduction in Arabidopsis thaliana and related Brassicaceae species. Proc Natl Acad Sci USA 2006, 103:5224-5229.

62. Schranz ME, Windsor AJ, Song B, Lawton-Rauh A, Mitchell-Olds T: Comparative Genetic Mapping in Boechera stricta, a Close Relative of Arabidopsis. Plant Physiol 2007, 144:286-298.

63. Stacey G, VandenBosch K: "Translational" legume biology. Models to crops. Plant Physiol 2005, 137:1173.

64. Koebener RMD, Varshney RK: Development and application of genomic models for large-crop plant genomes. In Model Plants and Crop Improvement. Edited by Varshney RK, Robert MD. Koebner. Boca Raton: FL CRC Press; 2006:1-9.

65. Broman KW, Wu H, Sen S, Churchill GA: R/qtl: QTL mapping in experimental crosses. Bioinformatics 2003, 19:889-890.

66. Broman KW, Sen S: A Guide to QTL Mapping with R/atl. New York: Springer; 2009.

67. Manichaikul A, Moon JY, Sen S, Yandell BS, Broman KW: A model selection approach for the identification of quantitative trait loci in experimental crosses, allowing epistasis. Genetics 2009, 181:1077-1086.

68. Bell CJ, Ecker J: Assignment of 30 microsatellite loci to the linkage map of Arabidopsis. Genomics 1994, 19:137-144.

69. Symonds W, Lloyd AM: An analysis of microsatellite loci in Arabidopsis thaliana: mutational dynamics and application. Genetics 2003, 165:1475-1488. 
70. Rumble SM, Lacroute P, Dalca AV, Fiume M, Sidow A, Brudno M: SHRiMP: Accurate mapping of short color-space reads. PLoS Comput Biol 2009, 5:e1000386.

71. Lamesch P, Berardini TZ, Li D, Swarbreck D, Wilks C, Sasidharan R, Muller R, Dreher K, Alexander DL, Garcia-Hernandez M, Karthikeyan AS, Lee CH, Nelson WD, Ploetz L, Singh S, Wensel A, Huala E: The Arabidopsis Information Resource (TAIR): improved gene annotation and new tools. Nucleic Acids Res 2012, 40:D1201-D1210.

doi:10.1186/1471-2164-14-655

Cite this article as: Fletcher et al:: Development of a next-generation NIL library in Arabidopsis thaliana for dissecting complex traits. BMC

Genomics 2013 14:655.

\section{Submit your next manuscript to BioMed Central and take full advantage of:}

- Convenient online submission

- Thorough peer review

- No space constraints or color figure charges

- Immediate publication on acceptance

- Inclusion in PubMed, CAS, Scopus and Google Scholar

- Research which is freely available for redistribution 